Introduction Rheumatoid arthritis (RA) and psoriatic arthritis (PsA) are both rheumatic autoimmune diseases, which share a number of similarities but also display differences. For example, the anti-IL17A biologic secukinumab turned out to be more effective in PsA than in RA. The anti-TNF biologic adalimumab on the other hand is equally indicated for both diseases. Synovial fibroblasts (SF) are one of the key effector cell types in the pathophysiology of RA and PsA.

Objectives We therefore investigated whether the effect of the two cytokines IL-17A and TNF- $\alpha$ as well as the effect of their corresponding biologics differs between RASF and PsASF, thus contributing to the difference seen in the therapeutic response. The effect of the IL-17A homolog IL-17F was also analyzed.

Methods SF were isolated from patients with PsA or RA, each undergoing surgery. SF from RA and PsA patients were stimulated with recombinant IL-17A, IL-17F and TNF- $\alpha$ alone or with respective combinations. Dose-response curve analysis was performed with IL-17A. The biologics secukinumab and adalimumab were used to block the effects on the SF. As a measure of the proinflammatory response, secretion of the cytokine IL-6 was quantified using an immunoassay.

Results RASF as well as PsASF responded to IL-17A (IL-17A: 13.7-fold vs 6.9-fold; $\mathrm{n}=3$ ), while IL-17F alone caused no induction of IL-6 secretion in either SF type. However, when used in combination with TNF- $\alpha$, both IL-17 isoforms, IL-17A and IL-17F, increased IL-6 secretion due to a strong synergistic effect with TNF- $\alpha$. Surprisingly, these effects were notably stronger for RASF than for PsASF (IL-17A: 544-fold vs 127fold, IL-17F: 54-fold vs 27-fold; $\mathrm{n}=3$ ). However, adalimumab and secukinumab were similarly effective in abolishing the synergistic effect of IL-17A and TNF- $\alpha$ in RASF as well as PsASF. Conclusions SF appear not to contribute to the differences in the therapeutic effectiveness of the anti-IL17A biologic secukinumab as the response to IL-17A alone and IL-17A together with TNF $-\alpha$ is not stronger for PsASF than for RASF. Furthermore, secukinumab was similarly effective for both SF types. The data also suggest that in a proinflammatory milieu with increased TNF levels IL-17A as well as IL-17F play a role in the SF-mediated pathophysiology of PsA and, therefore, approaches targeting TNF are effective in both diseases.

Acknowledgements Supported by an unrestricted educational grant from Celgene $\mathrm{GmbH}$.

Disclosure of Interest K. Frommer Grant/research support from: unrestricted educational grant from Celgene $\mathrm{GmbH}$, S. Rehart: None declared, U. Müller-Ladner: None declared, E. Neumann: None declared.

\section{P092/015 SYNOVIAL DERIVED TH17-LYMPHOCYTES FROM PATIENTS WITH JUVENILE IDIOPATHIC ARTHRITIS INDUCE CARTILAGE DEGRADATION BY SYNOVIAL FIBROBLASTS MEDIATED BY MMP9 IN AN EXPERIMENTAL ANIMAL MODEL}

${ }^{1} \mathrm{~T}$ Giani* ${ }^{*}{ }^{2} \mathrm{~F}$ Margheri, ${ }^{2} \mathrm{M}$ Del Rosso, ${ }^{3} \mathrm{~L}$ Maggi, ${ }^{3} \mathrm{~F}$ Annunziato, ${ }^{4} \mathrm{R}$ Cimaz. ${ }^{1}$ Rheumatology Unit, University of Florence and Siena; ${ }^{2}$ Department of Experimental and Clinical Biomedical Sciences 'Mario Serio'; ${ }^{3}$ Department of Experimental and Clinical Medicine; ${ }^{4}$ Rheumatology Unit, University of Florence, Florence, Italy

\subsection{6/annrheumdis-2018-EWRR2019.81}

Career situation of first and presenting author Student for a master or a $\mathrm{PhD}$.

Introduction The synovial fluid of Juvenile idiopathic Arthritis (JIA) is rich of Th17 and of Th-17-derived CD4+ CD161+ cells, also called non-classic-Th1. How such subpopulations drive the JIA joint damage is still a subject of great interest especially in light of the possible use of biological drugs able to selectively inhibit the activity of specific cytokines.

Objectives To clarify the role of Th17 and non-classical Th1 lymphocytes in the pathogenesis of joint cartilage destruction by synovial fibroblasts (SFbs).

Methods The role of different subsets of CD4+T cells was observed in the activation of SFbs in terms of cartilage degradation by normal and JIA SFbs and induction of proteases both in vitro and in vivo, using a SCID Mouse model through the 'inverse wrap' implantation technique.

Results JIA SFbs produce large amounts of MMP9 and efficiently degrade fragments of human cartilage wrapped in a collagen matrix containing the fibroblasts themselves and grafted under-skin on SCID mice (the 'inverse wrap model'). Similar effects were observed with SFbs of healthy subjects incubated with conditioned media of Th17 and of non-classic Th1. We shown that Th17 induce MMP9 in SFbs, while nonclassic Th1 act mainly by inducing urokinase-plasminogen-activator over-activity.

Conclusions IL-17 triggers the pathogenic chain leading to joint damage in patients with JIA.

\section{REFERENCES}

1. Maggi $L$, et al. T cell subpopulations in juvenile idiopathis arthritis and their modifications after biotherapies. Autoimmun Rev 2016;15:1141-44.

2. Nistala K, et al. Th17 plasticity in human autoimmune arthritis is driven by the inflammatory environment. Proc Natl Acad Sci U S A 2010;107:14751-6.

3. Miossec P, Korn T, Kuchroo VK. Interleukin-17 and type 17 helper T cells. N Engl J Med. 2009 August 27;361:888-98.

4. Maggi $L$, et al. Judex $M$, et al. 'Inverse wrap': an improved implantation technique for virus transduced synovial fibroblasts in the SCID mouse model forrheumatoid arthritis. Mod Rheumatol 2001;11:145-50.

5. Del Rosso $M$, et al. Multiple pathways of cell invasion are regulated by multiple families of serine proteases. Clin Exp Metastasis 2002;19:193-207.

6. Beringer A, Noack M, Miossec P. L-17 in Chronic Inflammation: From Discovery to Targeting. Trends Mol Med 2016 March;22:230-241.

7. Miossec P. Update on interleukin-17: a role in the pathogenesis of inflammatory arthritis and implication for clinical practice. RMD Open 2017 February 15;3.

Disclosure of Interest None declared.

\section{P093/016 IL-36 SIGNALING IN KERATINOCYTES IS MANDATORY IN IMIQUIMOD-INDUCED PSORIASIS IN MICE}

J Goldstein*, EY Bassoy, J Palomo, E Rodriguez, C Gabay. Department of Pathology and Immunology, Centre Médical Universitaire, Genève, Switzerland

\subsection{6/annrheumdis-2018-EWRR2019.82}

Career situation of first and presenting author Post-doctoral fellow.

Introduction A large body of evidence indicates that IL-36R signaling plays an important role in the pathogenesis of psoriasis. For instance, IL-36 receptor (IL-36R)-deficient mice showed impaired development of imiquimod (IMQ)-induced psoriasis, but the cells in which IL-36R signaling is involved have not been identified.

Objectives To assess the role of IL-36R signaling in keratinocytes in the development of IMQ-induced psoriasis.

Methods We constructed an IL-36R $\mathrm{R}^{\mathrm{fl} / \mathrm{fl}}$ mouse line in which exons 1 and 2 from the Il36r gene are flanked by loxP sites. Crossing these mice with $\mathrm{K} 5$-Cre transgenic mice induced a progeny with a specific deletion of $I l 36 r$ in keratinocytes (IL$36 \mathrm{R}^{\Delta \mathrm{K}}$ ). The selectivity of $I l 36 r$ gene targeting in keratinocytes was confirmed by RT-qPCR and ex vivo cell stimulation by 